\title{
Impact of the mesh fixation technique on operation time in laparoscopic repair of ventral hernias
}

\author{
E. B. Wassenaar · J. T. F. J. Raymakers $\cdot$ S. Rakic
}

Received: 18 January 2007 / Accepted: 6 July 2007 / Published online: 1 August 2007

(c) Springer-Verlag 2007

\begin{abstract}
Background Fixation of the prosthesis is one of the critical components of laparoscopic repair of ventral and incisional hernia (LRVIH). The impact of the fixation technique used on operative time has never been analyzed. We compared the duration of the operation according to the fixation technique used in a series of 138 patients with primary umbilical hernia.

Methods All patients underwent a straightforward repair by using completely standardized techniques. One hundred and seven patients had mesh fixation with a single crown of tackers (ProTack ${ }^{\circledR}$, TycoUSS, Norwalk, CT) and eight transabdominal sutures (TAS). Thirty-one patients had mesh fixation with a double crown of tackers (DC) without TAS.

Results There were no significant differences in age, sex, hospital stay, and morbidity between the two groups. Mean operating time for the technique with TAS was $50.6 \mathrm{~min}$ compared to $41.4 \mathrm{~min}$ for the DC technique. The mean difference in operating time was $9.2 \mathrm{~min}$. This difference was significant $(P=0.002)$. During a mean follow-up of 26.4 months, there were no recurrences in the entire series.

Conclusions The difference in operative times between the two operative techniques can be entirely accounted to the difference in the time needed for insertion of eight TAS as compared to the time needed for application of an inner crown of tackers. This strongly indicates that insertion of every single TAS prolongs LRVIH for approximately $1 \mathrm{~min}$. As long as no significant differences between the two fixation techniques are demonstrated on issues of
\end{abstract}

E. B. Wassenaar $(\varangle)$ · J. T. F. J. Raymakers · S. Rakic Department of Surgery, Twenteborg Hospital, P.O. Box 7600, 7600 SZ Almelo, The Netherlands

e-mail: ebwassenaar@zonnet.nl recurrence, complications, and postoperative pain, the time difference we have measured might be an argument in favor of the DC technique, especially when mesh fixation would require a large number of TAS.

Keywords Laparoscopy $\cdot$ Study, time $\cdot$ Surgical mesh · Surgical fixation devices $\cdot$ Hernia $\cdot$ Umbilical

\section{Introduction}

Laparoscopic repair of ventral and incisional hernia (LRVIH) is gaining increasing popularity due to its low recurrence rate, short hospital stay, and low complication rate. Fixation of the prosthesis is one of the critical components of LRVIH. Currently, the two most popular methods of mesh fixation are the use of helical tackers either with or without transabdominal sutures (TAS). Overall it appears that the two laparoscopic mesh fixation techniques are similar in outcomes [1]. However, the impact of the applied fixation technique on operative time has never been analyzed. We compared the duration of the operation according to the fixation technique used in a series of patients with primary umbilical hernia (PUH) who underwent a straightforward repair by using completely standardized techniques.

\section{Patients and methods}

Data were collected from operative reports of all 167 patients who underwent laparoscopic repair of PUH up to January 2007. To make the data more homogenous, we excluded patients who required adhesiolysis $(n=13)$ or simultaneously underwent another procedure $(n=12)$, or 
with whom a minor complication occurred intraoperatively, i.e., bleeding or equipment problem $(n=4)$. The remaining 138 patients underwent straightforward repair of PUH by using a completely standardized technique, and they represented the study group used to compare operative times between the two mesh fixation techniques.

Pneumoperitoneum was established by using a Veress needle. Three trocars $(10,5$, and $5 \mathrm{~mm})$ were inserted left laterally. A 1-mm-thick expanded polytetrafluoroethylene mesh (DualMesh ${ }^{\circledR}$, WL Gore, Flagstaff, AZ) of 15 by $10 \mathrm{~cm}$ was used to overlap the hernia opening by at least 3$4 \mathrm{~cm}$. The mesh was fixed using one of the following two techniques: either (1) with tackers (ProTack ${ }^{\circledR}$, TycoUSS, Norwalk, CT) along the periphery of the mesh at intervals of $1-1.5 \mathrm{~cm}$ and eight TAS placed equidistant also along the periphery ( $n=107$, further called the TAS technique) or (2) with a double ring of tackers alone without the use of TAS ( $n=31$, further called the DC technique). With this technique, also known as a "double crown technique" [2], the outer ring of tackers is the same as in the TAS technique. The inner ring of tackers is placed around the hernia opening about $1 \mathrm{~cm}$ apart. Transabdominal sutures were pulled through the abdominal wall with a suture passer (Gore Suture Passer Instrument ${ }^{\circledR}$, WLGore, Flagstaff, AZ). In the first 84 patients, the method of mesh fixation was based on the surgeon's preference. For the last 54 patients, the mesh fixation technique performed was randomly chosen in conjunction with another study being done at our hospital that compares postoperative pain following these two methods of mesh fixation. Once the fixation was completed, the abdomen was desufflated and the trocar site of $10 \mathrm{~mm}$ and skin were closed. The time taken from the stab wound for insertion of a Veress needle to completion of skin closure was recorded to the nearest minute.

Statistical analysis was done using the $t$ test. Significance was set at a $P<0.05$.

\section{Results}

Of the 138 patients in our study group, 106 were males and 36 females. Mean age was $55.0 \pm 12.3$ years. The TAS and DC groups were found to be well matched for sex and age. Mean operating time for the TAS technique was $50.6 \mathrm{~min}$ compared to $41.4 \mathrm{~min}$ for the DC technique. Mean difference in operating time was $9.2 \mathrm{~min}$. This difference was significant $(P=0.002)$.

Mean postoperative hospital stay was identical for both groups and equaled 1 day. Postoperative complications included seroma lasting longer than 6 weeks in seven patients and chronic pain at TAS sites in one patient. All seromas resolved without intervention. The patient with persisting pain underwent relaparoscopy and removal of all eight TAS that provided complete pain relief. There were no significant differences in morbidity between the two groups. No recurrences were detected during a mean follow-up of $26.4 \pm 25.6$ months.

\section{Discussion}

Besides prosthetic material that must sufficiently overlap the hernia defect, a reliable fixation of the prosthesis against the abdominal wall is a crucial component for success of LRVIH. Since the early years of LRVIH, there has been a strong belief among pioneers of this technique that reliable fixation of the mesh can only be achieved with the TAS technique $[3,4]$ and, in addition, that sutured mesh fixation is an imperative in LRVIH [5]. Another technique of fixation introduced later and consisting of a double crown of tackers only has been gaining increasing popularity due to a few specified advantages: technical simplicity, fewer incisions in the skin and possibly shorter operative time $[2,6]$. One of the most interesting issues on LRVIH currently being debated is which of these two techniques is better. Prospective randomized studies comparing the two techniques are missing. Meta-analysis of published literature indicates that the two laparoscopic mesh fixation techniques are similar in main outcome parameters such as recurrence and complication rate [1]. In the same analysis, the use of tackers alone resulted in a slightly shorter operative time than when the TAS technique was used. However, in most of the studies that were included in this meta-analysis, fixation with tackers was performed by applying only a single row of tackers or technical details of the tacker fixation technique were not explained. Reliable data comparing TAS technique and DC technique are definitely missing.

We decided to compare the two techniques in a maximally homogenous model of the procedure: same site of hernia, same logistics of the operation, same prosthetic material, same fixation device, and same operation technique until the moment that the outer ring of tackers is completed. The only difference between the two techniques was the completion of the mesh fixation. In one technique eight TAS had to be inserted and in the other technique usually 6-8 tackers of the inner crown. Once the fixation had been completed, whatever the method was, the rest of the procedure was again identical. Obviously, the difference in operative times between the two operative techniques can be entirely accounted to the difference in time needed for insertion of eight TAS compared to the time needed for application of an inner crown of tackers. Since tacking of the inner crown takes definitely not more than $1 \mathrm{~min}$ in laparoscopic repair of PUH, insertion of eight TAS required at least $8 \mathrm{~min}$. This strongly indicates that insertion of every single TAS prolongs LRVIH by approximately $1 \mathrm{~min}$. 
A laparoscopic repair of PUH is definitely the least complex procedure among all LRVIHs. Insertion of TAS is probably easier than in other LRIVHs due to a central location of the hernia, a general absence of adhesions, maximal space between the distended abdominal wall and the bowel underneath, and an excellent view. It may be anticipated that insertion of TAS during more complex laparoscopic repairs of incisional hernias at less suitable sites, in the presence of adhesions and proximity of the bowel can be much more challenging and as a consequence will require more time than during repair of a PUH.

Our results indicate that LRVIH by using the DC technique indeed requires less operative time than when the TAS technique is used. As long as no significant differences between the two fixation techniques are demonstrated on issues of recurrence, complications, and postoperative pain, the time difference we have measured might be an argument in favor of the DC technique, especially when mesh fixation would require a large number of TAS.

\section{References}

1. Rudmik LR, Schieman C, Dixon E, Debru E (2006) Laparoscopic incisional hernia repair: a review of the literature. Hernia 10:110 119

2. Morales-Conde S, Morales-Mendez S (2003) Hernioplasty with the double crown technique. In: LeBlanc KA (ed) Laparoscopic hernia surgery. Arnold, London, pp 133-142

3. Heniford BT, Park A, Ramshaw BJ, Voeller G (2003) Laparoscopic repair of ventral hernias: 9 years' experience with 850 consecutive hernias. Ann Surg 238: 391-399 discussion 399-400

4. LeBlanc KA, Whitaker JM, Bellanger DE, Rhynes VK (2003) Laparoscopic incisional and ventral hernioplasty: lessons learned from 200 patients. Hernia 7:118-124

5. Adrales GL, Park AE (2003) Technological and instrumentation aspects of laparoscopic hernia surgery. In: leBlanc KA (ed) Laparoscopic hernia surgery. Arnold, London, pp 7-15

6. Carbajo MA, Martp del Olmo JC, Blanco JI, Toledano M, de la Cuesta C, Ferreras C, Vaquero C (2003) Laparoscopic approach to incisional hernia. Surg Endosc 17:118-122 